\title{
ON CENTRALISERS AND NORMALISERS FOR GROUPS
}

\author{
BORIS ŠIROLA
}

(Received 28 November 2011; accepted 31 May 2012)

\begin{abstract}
Let $\mathbb{K}$ be a field, char $(\mathbb{K}) \neq 2$, and $G$ a subgroup of $\mathrm{GL}(n, \mathbb{K})$. Suppose $g \mapsto g^{\sharp}$ is a $\mathbb{K}$-linear antiautomorphism of $G$, and then define $G_{1}=\left\{g \in G \mid g^{\sharp} g=I\right\}$. For $C$ being the centraliser $C_{G}\left(G_{1}\right)$, or any subgroup of the centre $\mathcal{Z}(G)$, define $G^{(C)}=\left\{g \in G \mid g^{\sharp} g \in C\right\}$. We show that $G^{(C)}$ is a subgroup of $G$, and study its structure. When $C=C_{G}\left(G_{1}\right)$, we have that $G^{(C)}=\mathcal{N}_{G}\left(G_{1}\right)$, the normaliser of $G_{1}$ in $G$. Suppose $\mathbb{K}$ is algebraically closed, $C_{G}\left(G_{1}\right)$ consists of scalar matrices and $G_{1}$ is a connected subgroup of an affine group $G$. Under the latter assumptions, $\mathcal{N}_{G}\left(G_{1}\right)$ is a self-normalising subgroup of $G$. This holds for a number of interesting pairs $\left(G, G_{1}\right)$; in particular, for those that we call parabolic pairs. As well, for a certain specific setting we generalise a standard result about centres of Borel subgroups.
\end{abstract}

2010 Mathematics subject classification: primary 20E34; secondary 20G15.

Keywords and phrases: centraliser, normaliser, self-normalising subgroup, parabolic subgroup.

\section{Introduction}

Unless specified otherwise, throughout this paper $\mathbb{K}$ always denotes a field of characteristic $\neq 2$, and $\overline{\mathbb{K}}$ its fixed algebraic closure. We denote by $\boldsymbol{\mu}_{l}(\mathbb{K})$ the group of $l$ th roots of unity in $\mathbb{K}$. We denote by $\mathbb{F}_{p}$ the finite field with $p$ elements. Given a group $G$, we denote by $\mathcal{Z}(G)$ its centre. For a subgroup $G_{1}$ of $G$, we denote by $C_{G}\left(G_{1}\right)$ and $\mathcal{N}_{G}\left(G_{1}\right)$ the centraliser and normaliser of $G_{1}$ in $G$, respectively. The letter $I$ is reserved for the identity matrix of some $\operatorname{GL}(n, \mathbb{K})$. We often identify a scalar $\lambda \in \mathbb{K}^{\times}$ and the scalar matrix $\lambda I$. Under this identification we consider $\mathbb{K}^{\times}$as a subgroup of $\mathrm{GL}(n, \mathbb{K})$.

An interesting problem in group theory and representation theory is to study the normalisers $\mathcal{N}_{G}\left(G_{1}\right)$ for various pairs of groups $\left(G, G_{1}\right)$, where $G_{1} \leq G$. In particular, it is worth knowing for which pairs $\left(G, G_{1}\right)$ the answer to the following question is affirmative; see Remark 1.3.

Question 1.1. Is $\mathcal{N}_{G}\left(G_{1}\right)$ self-normalising in $G$ ?

The author was supported in part by the Ministry of Science, Education and Sports, Republic of Croatia, Grant No. 900-194134.

(C) 2012 Australian Mathematical Publishing Association Inc. 0004-9727/2012 \$16.00 
A related problem is to study the class of all self-normalising subgroups of a given $G$. As one famous example of this let us mention the Chevalley normaliser theorem, which states that every parabolic subgroup of a connected affine group is both self-normalising and connected; see, for example, [Bor, Theorem 11.16].

Now we would like to be more precise about the pairs $\left(G, G_{1}\right)$ that we will study. Let $\boldsymbol{G}$ be a closed subgroup of $\operatorname{GL}(m, \overline{\mathbb{K}})$. Put $G=\boldsymbol{G}(\mathbb{K})$, the group of $\mathbb{K}$-rational points of $\boldsymbol{G}$. We want to consider some interesting subgroups $G_{1} \leq G$, and obtain some useful information concerning the normaliser $\mathcal{N}_{G}\left(G_{1}\right)$. As will be seen below, these normalisers are often self-normalising subgroups of $G$. We should also say here that our subgroups $G_{1}$ will in fact always be the groups of $\mathbb{K}$-points $\boldsymbol{G}_{1}(\mathbb{K})$, for certain algebraic $\mathbb{K}$-subgroups $\boldsymbol{G}_{1} \leq \boldsymbol{G}$. More precisely, our main interest is when $\boldsymbol{G}$ and $\boldsymbol{G}_{1}$ are both either reductive or parabolic groups. As is well known, these two kinds of algebraic groups, and the corresponding groups of their rational points, play fundamental roles in representation theory. As another fact worthy of mention, we also have the following: given a pair of algebraic groups $\left(\boldsymbol{G}, \boldsymbol{G}_{1}\right)$, both of which are connected, the normaliser $\mathcal{N}_{\boldsymbol{G}}\left(\boldsymbol{G}_{1}\right)$ very often will not be connected; see Proposition 3.10. This fact makes our study more interesting and complicated.

Given $G$, for various reasons it will be helpful to study certain 'big' subgroups $G_{1}$. For example, then we can expect to have good control over the restrictions of some special $G$-representations to $G_{1}$. For a recent work which explains why it would be useful to understand the normaliser $\mathrm{N}=\mathcal{N}_{G}\left(G_{1}\right)$, when $G$ is a (complex) reductive group and $G_{1}$ is its reductive subgroup, see [Ko3, Section 2]; see also [BK, Ko1, Ko2, $\mathrm{Ks}, \mathrm{LS}]$ for some related results worth knowing. Notice that, for $G$ and $G_{1}$ as above, $\mathrm{N}$ is often again reductive, and so one can repeat the procedure by computing $\mathcal{N}_{G}(\mathrm{~N})$. But our theorem below states that in many interesting situations one has $N=\mathcal{N}_{G}(\mathrm{~N})$. Concerning the 'big' subgroups mentioned, we would like to emphasise that we are also interested in the opposite situation-that is, when $G_{1}$ is a 'small' subgroup of $G$. It turns out that for a number of such pairs $\left(G, G_{1}\right)$ we can describe the structure of the corresponding normalisers $\mathcal{N}_{G}\left(G_{1}\right)$. For certain reasons why this should be so, and some basic examples as well, we refer the reader to [S2] and [S3, Example 1.7]; see also [01, O2]. Another related fact worth mentioning here is that distinguished nilpotent orbits are characterised by being self-normalising. More precisely, a threedimensional subalgebra $\mathfrak{s}$ of a (complex) semisimple Lie algebra $\mathfrak{g}$ is self-normalising if and only if it is distinguished (as shown in a paper of this author in preparation).

Let us now present the content of this paper, and make some comments. Suppose that $G \leq \mathrm{GL}(n, \mathbb{K})$ is a group having an antiautomorphism $\delta: g \mapsto g^{\sharp}$. Then define

$$
G_{1}=G^{(I)}=\left\{g \in G \mid g^{\sharp} g=I\right\},
$$

a subgroup of $G$. For $C$ being $C_{G}\left(G_{1}\right)$, or an arbitrary subgroup of $\mathcal{Z}(G)$, define a set

$$
G^{(C)}=\left\{g \in G \mid g^{\sharp} g \in C\right\} ;
$$

note that, under the latter notation, $G_{1}=G^{(\{I\})}$. Here the above map $\delta$ will always be the restriction to $G$ of some $\mathbb{K}$-endomorphism of $M_{n}(\mathbb{K})$, which we also denote by $\delta$. Let us 
also emphasise that in a number of interesting situations we will have $C_{G}\left(G_{1}\right)=\mathcal{Z}(G)$; in particular, the last equality will obviously hold if the assumption $C_{\boldsymbol{G}}\left(\boldsymbol{G}_{1}\right) \subseteq \mathbb{K}^{\times}$, from the theorem below, also holds. Now we are ready to state that theorem, which is our first main result. Provided we work with algebraic groups, it is a generalisation of the corresponding results in [S3, S4].

THEOREM 1.2. If $C=C_{G}\left(G_{1}\right)$, then

$$
\mathcal{N}_{G}\left(G_{1}\right)=G^{(C)}=\left\{g \in G \mid g^{\sharp} g \in C_{G}\left(G_{1}\right)\right\} .
$$

Suppose, in particular, that the base field $\mathbb{K}$ is algebraically closed, and that $\boldsymbol{G}_{1}=\boldsymbol{G}^{(I)}$ is a connected algebraic $\mathbb{K}$-subgroup of an affine group $\boldsymbol{G} \leq \mathrm{GL}(n, \mathbb{K})$. Suppose also that the centraliser $C_{\boldsymbol{G}}\left(\boldsymbol{G}_{1}\right)$ is a subgroup of $\mathbb{K}^{\times}$. Then the normaliser $\mathcal{N}_{\boldsymbol{G}}\left(\boldsymbol{G}_{1}\right)$ is a self-normalising subgroup of $\boldsymbol{G}$.

In Section 3 we prove the above theorem, as well as several useful preparatory facts about $G^{(C)}$, that are gathered in Theorem 3.2.

Remark 1.3. Question 1.1 is much more difficult if we work with groups of rational points. More precisely, suppose that $\mathbb{K}, \boldsymbol{G}$ and $\boldsymbol{G}_{1}$ are as in the above theorem, and let $\mathbb{F} \subseteq \mathbb{K}$ be a subfield. Consider the corresponding groups of $\mathbb{F}$-points $G=\boldsymbol{G}(\mathbb{F})$ and $G_{1}=G_{1}(\mathbb{F})$. It seems quite complicated to say in general for which $\mathbb{F}$ the answer to Question 1.1 is affirmative; see Remark 3.8.

There are many pairs of groups $\left(G, G_{1}\right)$, and pairs of the corresponding Lie algebras $\left(\mathfrak{g}, \mathfrak{g}_{1}\right)$, for which the normalisers $\mathcal{N}_{G}\left(G_{1}\right), \mathcal{N}_{\mathfrak{g}}\left(\mathfrak{g}_{1}\right)$ and $\mathcal{N}_{G}\left(\mathfrak{g}_{1}\right)$ can be computed. For examples of various pairs $\left(G, G_{1}\right)$ and $\left(\mathfrak{g}, \mathfrak{g}_{1}\right)$, where $\left(G, G_{1}\right)$ are groups of rational points of some reductive algebraic groups $\left(\boldsymbol{G}, \boldsymbol{G}_{1}\right)$, see [S1, S2, S3, S4]. Such pairs might be called reductive pairs. The second goal of this paper is to point out that we also have a number of nonreductive pairs $\left(G, G_{1}\right)$. That is, we show in Section 4 that there are many pairs which might be called parabolic pairs. More precisely, there we consider $G=\operatorname{SL}(2 n, \mathbb{K})$ or $\operatorname{GL}(2 n, \mathbb{K})$, and a certain antiautomorphism $A \mapsto A^{\sharp}$ of $G$ which then gives the corresponding subgroup $G_{1}$ of $G$. We have the following proposition which shows, in particular, that the pair $\left(Q, Q_{1}\right)$, defined below, fits into the setting of our theorem; for a slightly more general claim, see Proposition 4.3.

Proposition 1.4. Let $\mathbb{K}$ be a field such that char $(\mathbb{K}) \neq 2$, and which is different from $\mathbb{F}_{3}$. Let $Q$ be any $\sharp$-stable standard parabolic subgroup of $G$, and $Q_{1}=Q \cap G_{1}$ a parabolic subgroup of $G_{1}$. Then

$$
C_{Q}\left(Q_{1}\right)=\mathcal{Z}(G)= \begin{cases}\mu_{2 n}(\mathbb{K}) & \text { if } G=\operatorname{SL}(2 n, \mathbb{K}), \\ \mathbb{K}^{\times} & \text {if } G=\operatorname{GL}(2 n, \mathbb{K}) ;\end{cases}
$$

and therefore, in particular, $\mathcal{N}_{Q}\left(Q_{1}\right)=\left\{g \in Q \mid g^{\sharp} g \in \mathcal{Z}(G)\right\}$.

Proposition 4.3, together with Proposition 4.2, might be understood as a technical result that in a specific setting generalises a standard fact about centres of Borel subgroups; see Lemma 4.1. 


\section{Preliminaries}

2.1. Pairs $\left(\boldsymbol{G}, \boldsymbol{G}_{\mathbf{1}}\right)$. First we briefly explain what kind of groups $G$ and their subgroups $G_{1}$ we are interested in; more details on what follows can be found in [S3, S4]. For that purpose we will consider certain $\mathbb{K}$-linear endomorphisms $A \mapsto A^{\sharp}$ of $M_{n}(\mathbb{K})$ satisfying the following:

(甘1) $I^{\sharp}=I$;

$(\sharp 2)(A B)^{\sharp}=B^{\sharp} A^{\sharp}$, for all $A, B \in M_{n}(\mathbb{K})$.

For later applications it is useful to note that $(\sharp \mathbf{1})$ and $(\sharp 2)$ imply the following:

(\#3) $\left(A^{-1}\right)^{\sharp}=\left(A^{\sharp}\right)^{-1}$, for all $A \in \mathrm{GL}(n, \mathbb{K})$.

Let us emphasise that there will be a number of $\mathbb{K}$-endomorphisms $\delta: A \mapsto A^{\sharp}$ of a concrete group $G$; a more precise statement can be found in [S3, Remark 1.2].

Let $G \leq \mathrm{GL}(n, \mathbb{K})$ be any group. Suppose we have a $\mathbb{K}$-endomorphism $A \mapsto A^{\sharp}$ satisfying ( $\sharp \mathbf{1})$ and $(\sharp 2)$. Next suppose that $G$ is $\sharp$-stable; that is, $g^{\sharp} \in G$, for all $g \in G$. Then define $G_{1}$ by (1.1).

2.2. Two technical conditions. For later use let us formulate the following two technical conditions. In order to justify why we introduce them, the reader may consult [S3, Corollary 0.2] and Section 3 below; see also Remark 2.4. Here $G$ is again a subgroup of some $\operatorname{GL}(n, \mathbb{K})$, while $C \leq \mathcal{Z}(G)$.

Condition $(\diamond)$. There is $\omega \in C \backslash C^{2}$ for which we can find $W \in G$ so that $W^{\sharp} W=\omega$.

Condition $(\triangle)$. Condition $(\diamond)$ holds, and for $\omega$ as specified in it we have $C^{2} \cup \omega C^{2}=C$.

Let us emphasise that for $C$ finite and satisfying Condition $(\diamond)$ we automatically have Condition $(\triangle)$ fulfilled.

2.3. Remarks for $\boldsymbol{G}=\mathbf{S L}(\boldsymbol{m}, \mathbb{K})$. Let $m \in \mathbb{N}, m>2$, and $G=\operatorname{SL}(m, \mathbb{K})$. If $m=2 n$, define $J=J_{\varepsilon}^{\text {even }}$, and if $m=2 n+1$, define $J=J^{\text {odd }}$, where these block matrices are

$$
J_{\varepsilon}^{\text {even }}=\left(\begin{array}{cc}
0 & I \\
\varepsilon I & 0
\end{array}\right) \quad \text { and } \quad J^{\text {odd }}=\left(\begin{array}{cc}
1 & 0 \\
0 & J_{+}^{\text {even }}
\end{array}\right) ;
$$

$I$ is the identity matrix of size $n$, and $\varepsilon= \pm 1$. For $A \in M_{m}(\mathbb{K})$ define $A^{\sharp}=J^{t} A^{t} J$.

Lemma 2.1. The map $A \mapsto A^{\sharp}$ is a $\mathbb{K}$-linear involution of $M_{n}(\mathbb{K})$ satisfying conditions (\#1) and (\#2). Moreover, this map is an antiautomorphism of $G$.

Now define $G_{1}$ as in the Introduction; it turns out that $G_{1}=\operatorname{Sp}(m, \mathbb{K})$ or $\operatorname{SO}(m, \mathbb{K})$. The following is a weak version of the main result of [S4].

Theorem 2.2. Let $G=\mathrm{SL}(m, \mathbb{K}), G_{1}$ be as above, $\mathrm{C}=C_{G}\left(G_{1}\right)$ and $\mathrm{N}=\mathcal{N}_{G}\left(G_{1}\right)$.

(i) We have $\mathrm{C}=\boldsymbol{\mu}_{m}(\mathbb{K})$, and $\mathrm{C}^{2}=\boldsymbol{\mu}_{n}(\mathbb{K})$ for $m=2 n$, while $\mathrm{C}^{2}=\mathrm{C}$ for $m=2 n+1$.

(ii) We have

$$
\mathrm{N}=\left\{g \in G \mid g^{\sharp} g \in \mathrm{C}\right\},
$$

and this is a self-normalising subgroup of $G$. 
(iii) Define

$$
\mathrm{N}^{\prime}=\left\{g \in G \mid g^{\sharp} g \in \mathrm{C}^{2}\right\} .
$$

Then

$$
\mathrm{N}^{\prime} / G_{1} \cong \mathrm{C} \quad \text { and } \quad \begin{cases}\mathrm{N} / \mathrm{N}^{\prime} \cong \mu_{2}(\mathbb{K}) & \text { if Condition }(\diamond) \text { holds } \\ \mathrm{N}=\mathrm{N}^{\prime} & \text { otherwise }\end{cases}
$$

REMARK 2.3.

(a) Clearly, as for $m$ odd we have $\mathrm{C}^{2}=\mathrm{C}$, this time Condition $(\diamond)$ does not hold. So $\mathrm{N}=\mathrm{N}^{\prime}$, and assertion (iii) of the theorem reads as $\mathrm{N} / G_{1} \cong \mathrm{C}$.

(b) Note that now $\mathrm{C}$ is a finite group, and thus Condition $(\diamond)$ implies Condition $(\triangle)$.

(c) A similar result holds for $G=\mathrm{GL}(m, \mathbb{K})$, where then $\mathrm{C}=\mathbb{K}^{\times}$.

REMARK 2.4. Concerning the general problem on normalisers and self-normalising subgroups, we have obtained certain definite results in [S3, S4] and the present paper. But, as already emphasised in the two papers mentioned, a part of our research strongly depends on Condition ( $\diamond$ ) (for $n$ and $\mathbb{K}$ arbitrary), which seems to be quite delicate. In order to justify that condition it might be helpful to discuss briefly what happens for prime fields, provided that we work just in the special setting of the above theorem. Notice that then Condition $(\diamond)$ takes the following form: there exists $\omega \in \boldsymbol{\mu}_{2 n}(\mathbb{K}) \backslash \boldsymbol{\mu}_{n}(\mathbb{K})$ for which we can find some $W \in G$ satisfying $W^{\sharp} W=\omega I$.

First take $\mathbb{K}=\mathbb{Q}$. For $n$ even, $\boldsymbol{\mu}_{2 n}(\mathbb{Q})=\boldsymbol{\mu}_{n}(\mathbb{Q})=\{-1,1\}$, and so Condition $(\diamond)$ does not hold. On the other hand, for $n$ odd, $\boldsymbol{\mu}_{2 n}(\mathbb{Q}) \backslash \boldsymbol{\mu}_{n}(\mathbb{Q})=\{-1\}$. Thus, when $\varepsilon=1$ (that is, the orthogonal case), for $\omega=-1$ we can take $W=\left(\begin{array}{cc}0 & I \\ -I & 0\end{array}\right) \in \operatorname{SL}(2 n, \mathbb{Q})$. So, for these $\omega$ and $W$, Condition $(\diamond)$ holds. But when $\varepsilon=-1$ (that is, the symplectic case) things seem to be less clear. Namely, for $n$ odd satisfying $n \equiv 3(\bmod 4)$, Condition $(\diamond)$ does not hold; see [S3, Lemma 3.10] for a more general observation. But for $n \equiv 1(\bmod 4)$, we do not know whether we have $W \in \operatorname{SL}(2 n, \mathbb{Q})$ so that $W^{\sharp} W=-I$; however, we do believe that there is no such $W$.

Now consider $\mathbb{K}=\mathbb{F}_{p}$, where $p$ is an odd prime. If $\operatorname{gcd}(n, p-1)=1$, then one knows that $\boldsymbol{\mu}_{2 n}\left(\mathbb{F}_{p}\right)=\{-1,1\}$ and $\boldsymbol{\mu}_{n}\left(\mathbb{F}_{p}\right)=\{1\}$; see, for example, [Kob, Ch. II]. Thus, here one has to check whether there exists some $W \in \operatorname{SL}\left(2 n, \mathbb{F}_{p}\right)$ so that $W^{\sharp} W=-I$. On the other hand, consider the case $2 n=p-1$, for example. Then we have a primitive $2 n$th root of unity; that is, $\eta$ so that $\boldsymbol{\mu}_{2 n}\left(\mathbb{F}_{p}\right)=\left\{\eta, \eta^{2}, \ldots, \eta^{2 n}=1\right\}$. Hence we see that

$$
\boldsymbol{\mu}_{2 n}\left(\mathbb{F}_{p}\right) \backslash \boldsymbol{\mu}_{n}\left(\mathbb{F}_{p}\right)=\left\{\eta, \eta^{3}, \ldots, \eta^{2 n-1}\right\} .
$$

We should now check whether we have some odd power $\eta^{j}$ so that we can find for it some $W_{j} \in \operatorname{SL}\left(2 n, \mathbb{F}_{p}\right)$ satisfying $W_{j}^{\sharp} W_{j}=\eta^{j} I$. But all this seems to be a nontrivial combinatorial task.

\section{On the structure of $G^{(C)}$ and proof of Theorem 1.2}

Unless otherwise stated, throughout this section $G$ is a group having an antiautomorphism $g \mapsto g^{\sharp}$; we can take $G \leq \mathrm{GL}(n, \mathbb{K})$. Also, $C$ is a subgroup of $\mathcal{Z}(G)$. We begin with the following simple lemma. 
Lemma 3.1. The set $H=G^{(C)}$, defined by (1.2), is a subgroup of $G$.

Proof. First note that $g^{\sharp} g=g g^{\sharp}$, for $g \in H$. To see this, put $\gamma_{1}=g^{\sharp} g$ and $\gamma_{2}=g g^{\sharp}$. Then $g \gamma_{1}=\gamma_{2} g$. But as we have in particular $\gamma_{1} \in \mathcal{Z}(G)$, it clearly follows that $\gamma_{1}=\gamma_{2}$, as we had to show.

Suppose now that $g_{i} \in H$, and then denote $z_{i}=g_{i}^{\sharp} g_{i}$, for $i=1,2$. Then

$$
\left(g_{1} g_{2}^{-1}\right)^{\sharp} g_{1} g_{2}^{-1}=\left(g_{2}^{\sharp}\right)^{-1} z_{1} g_{2}^{-1}=z_{1}\left(g_{2} g_{2}^{\sharp}\right)^{-1}=z_{1} z_{2}^{-1} \in C ;
$$

where we use condition ( $\sharp 3)$ and the above fact. So, $H$ is indeed a subgroup of $G$.

At this point we introduce the following notation and assumptions. Let $H$ be as in the previous lemma. In particular, for $C=\{I\}$ we have a subgroup $G_{1}=G^{(I)}$ of $G$. Also, choose an endomorphism $\xi$ of $C$,

$$
C \ni x \mapsto \xi(x)=x^{2} \in C .
$$

Denote by $C^{2}$ its image, and then define a subgroup $H^{\prime}=G^{\left(C^{2}\right)}$ of $H$.

The first goal of this section is to study the structure of $G^{(C)}$. Concerning that, we have the following theorem. The first statement of its part (i) has already been proved via Lemma 3.1. The rest will be proved via Lemmas 3.3 and 3.5.

Theorem 3.2. Suppose that $G$ satisfies the condition

$$
\mathcal{Z}(G) \subseteq \mathbb{K}^{\times}
$$

(i) The set $G^{(C)}$ is a subgroup of $G$, and $G^{\left(C^{2}\right)}$ is a normal subgroup of $G^{(C)}$. If Condition $(\diamond)$ does not hold, then $G^{(C)}=G^{\left(C^{2}\right)}$. If Condition $(\triangle)$ holds, then $G^{(C)} / G^{\left(C^{2}\right)} \cong \mu_{2}(\mathbb{K})$.

(ii) We have

$$
G^{\left(C^{2}\right)} / G_{1} \cong C^{2}
$$

(iii) If Condition $(\triangle)$ holds, then

$$
G^{(C)} / G_{1} \cong C .
$$

As we will see, the above condition (3.1) on centres is a reasonable one; and it holds for a number of interesting groups $G$. Also, note that $\mathcal{Z}(G) \leq C_{G}(S)$, for any subgroup $S \leq G$.

The following lemma is in fact a more general version of the second half of [S3, Theorem 3.7(i)].

LEMMA 3.3.

(i) For a certain epimorphism $\sigma^{\prime}$, we have a short exact sequence

$$
1 \rightarrow G_{1} \hookrightarrow H^{\prime} \stackrel{\sigma^{\prime}}{\rightarrow} C^{2} \rightarrow 1
$$

In particular, if Condition $(\diamond)$ does not hold, then $H=H^{\prime}$.

(ii) Suppose that the group $C$ is finite, and Condition $(\diamond)$ holds. Then, for a certain extension $\sigma$ of $\sigma^{\prime}$, we have a short exact sequence of groups

$$
1 \rightarrow G_{1} \hookrightarrow H \stackrel{\sigma}{\rightarrow} C \rightarrow 1
$$


Proof. (ii) Assume that Condition $(\diamond)$ holds; note that now necessarily $-I \in C$ and $\operatorname{Ker} \xi=\{ \pm I\}$. For any $\lambda \in C$ define a subset

$$
\Gamma_{\lambda}=\left\{g \in G \mid g^{\sharp} g=\lambda\right\}
$$

of $G$; note that $\Gamma_{I}=G_{1}$. As for any $c \in C$ we have $c^{\sharp}=c$, it immediately follows that $c \in \Gamma_{c^{2}}$; here we use assumption (3.1). Thus, in particular, $\Gamma_{\lambda} \neq \emptyset$ for all $\lambda \in C^{2}$. Next we observe that the following are equivalent.

(a) Condition $(\diamond)$ holds.

(b) We have $\Gamma_{\lambda} \neq \emptyset$, for all $\lambda \in C$.

To see that $(\mathrm{a}) \Rightarrow(\mathrm{b})$ we first take $\omega$ and $W$ as in Condition $(\diamond)$. Then define a map $d: C^{2} \rightarrow C \backslash C^{2}, d(\lambda)=\omega \lambda$. It is clear that $d$ is well defined and injective. Furthermore, $C^{2} \cap \omega C^{2}=\emptyset$. Hence it immediately follows that

$$
C^{2} \cup \omega C^{2}=C,
$$

a disjoint union; here we use the fact that $C$ is finite. Therefore $d$ is surjective; that is, $d$ is bijective. Now take, for any $\lambda \in C^{2}$, some $U_{\lambda} \in \Gamma_{\lambda}$. Clearly, $W U_{\lambda} \in \Gamma_{d(\lambda)}$. Thus we have (b).

For any $\lambda \in C$, let $U_{\lambda} \in \Gamma_{\lambda}$ be arbitrary. Then it is easy to see that the map $\Xi_{\lambda}: G_{1} \rightarrow \Gamma_{\lambda}$, given as $\Xi_{\lambda}(g)=U_{\lambda} g$, is well defined and bijective. Hence it follows that we have a disjoint union

$$
H=\bigcup_{\lambda \in C} \Gamma_{\lambda}
$$

Next note that $\Gamma_{\lambda_{1}} \Gamma_{\lambda_{2}} \subseteq \Gamma_{\lambda_{1} \lambda_{2}}$, for any $\lambda_{1}, \lambda_{2} \in C$. Thus the map $\sigma: H \rightarrow C, \sigma_{\mid \Gamma_{\lambda}}=\lambda$, is a group epimorphism with the kernel $G_{1}$. This proves (ii).

(i) If Condition $(\diamond)$ does not hold, then $\Gamma_{\lambda}=\emptyset$, for all $\lambda \in C \backslash C^{2}$; and so $H^{\prime}=$ $\bigcup_{\lambda \in C^{2}} \Gamma_{\lambda}=H$. Now (i) follows easily.

Remark 3.4. (a) Note that part (ii) of the previous lemma holds for some infinite groups $C$ as well. But now we have to take as an assumption that (3.2) holds. Concerning this, consider a $\mathbb{K}$-endomorphism $A \mapsto A^{\sharp}$, of $M_{2}(\mathbb{K})$, given by $\left(\begin{array}{ll}x & y \\ z & t\end{array}\right)^{\sharp}=$ $\left(\begin{array}{ll}t & y \\ z & x\end{array}\right)$; see Section 2. Clearly, it satisfies $(\sharp 1)$ and $(\sharp 2)$. Also, $G=\operatorname{GL}(2, \mathbb{K})$ is $\sharp$-stable. Let $C=\mathcal{Z}(G)=\mathbb{K}^{\times}$. Both for $\mathbb{K}=\mathbb{Q}$ and $\mathbb{R}$ we have that Condition $(\diamond)$ holds. For example, for $\mathbb{Q}$ we can take $\omega=2$ and $W=\left(\begin{array}{ll}2 & 0 \\ 0 & 1\end{array}\right)$, and for $\mathbb{R}$ we can take $\omega=-1$ and $W=\left(\begin{array}{rr}-1 & 0 \\ 0 & 1\end{array}\right)$. But for $\mathbb{R}$ we have (3.2), while for $\mathbb{Q}$ we do not.

(b) Let $\mathbb{K}$ be any field of characteristic $\neq 2$, and $C=\boldsymbol{\mu}_{2 n}(\mathbb{K})$. If $n$ is odd, then $C \neq C^{2}=\mu_{n}(\mathbb{K})$; for example, $-1 \in C \backslash C^{2}$. But for $n$ even the last observation does not have to hold; for example, take $\mathbb{K}=\mathbb{R}$ and $n=2$.

Claim (ii) of the following lemma generalises a part of [S3, Corollary 3.8].

LEMMA 3.5.

(i) The subgroup $H^{\prime}$ is normal in $H$.

(ii) Suppose that both Condition ( $\diamond)$ and (3.2) hold. Then

$$
H / H^{\prime} \cong \mu_{2}(\mathbb{K}) \text {. }
$$


Proof. (i) Let $h \in H$ and $h_{0} \in H^{\prime}$ be arbitrary, and then define $g=h h_{0} h^{-1}$. As $h^{\sharp} h=$ $c \in C$ and $h_{0}^{\sharp} h_{0}=c_{0}^{2} \in C^{2}$, for some $c, c_{0} \in C$, it follows that $g^{\sharp} g=c_{0}^{2}$. This means that $g \in H^{\prime}$, as required.

(ii) Let $\omega$ and $W$ be as in Condition ( $\diamond)$. Suppose then that $g \in H \backslash H^{\prime}$, and define $h=W^{-1} g$. Provided that $g^{\sharp} g=\lambda \in C \backslash C^{2}$, we have $h^{\sharp} h=\lambda \omega^{-1}$. But (3.2) ensures that $\lambda \omega^{-1} \in C^{2}$, and so $h \in H^{\prime}$; that is, $g \in W H^{\prime}$. Thus $H=H^{\prime} \cup W H^{\prime}$. It is clear, moreover, that the latter union is disjoint. Thus the index $\left(H: H^{\prime}\right)=2$, and therefore the lemma follows. More precisely, the map $\tau: H \rightarrow \mu_{2}(\mathbb{K}), \tau_{\mid H^{\prime}}=1$ and $\tau_{\mid W H^{\prime}}=-1$, is a group epimorphism with the kernel $H^{\prime}$; here we use $W H^{\prime}=H^{\prime} W$ and $W^{2} \in H^{\prime}$.

The following simple lemma presents our crucial auxiliary observation, which is interesting in its own right.

Lemma 3.6. Let $G$ be an arbitrary group having an antiautomorphism $g \mapsto g^{\sharp}$, and then define $G_{1}$ as in (1.1). We have

$$
\mathcal{N}_{G}\left(G_{1}\right)=\left\{g \in G \mid g^{\sharp} g \in C_{G}\left(G_{1}\right)\right\} ;
$$

that is, by putting $C=C_{G}\left(G_{1}\right)$,

$$
\mathcal{N}_{G}\left(G_{1}\right)=G^{(C)} .
$$

Proof. Suppose that $g \in G^{(C)}$; that is, $g \in G$ satisfies

$$
g^{\sharp} g=c(g) \in C \text {. }
$$

Let then $x \in G_{1}$ be arbitrary, and define $y=y(x)=g x g^{-1}$. Using (3.3), we clearly have

$$
\begin{aligned}
y^{\sharp} y & =\left(g^{\sharp}\right)^{-1} x^{\sharp} c(g) x g^{-1}=\left(g^{\sharp}\right)^{-1} x^{\sharp} x c(g) g^{-1} \\
& =\left(g^{\sharp}\right)^{-1} c(g) g^{-1}=e ;
\end{aligned}
$$

here $e$ denotes the identity element of $G$. In other words, $y \in G_{1}$. Thus we have proved that $g \in \mathcal{N}_{G}\left(G_{1}\right)$; and, as a conclusion, $G^{(C)} \subseteq \mathcal{N}_{G}\left(G_{1}\right)$.

For the opposite inclusion, take any $g \in G$ so that the above defined $y=y(x) \in G_{1}$ for every $x \in G_{1}$. The last condition means that $y^{\sharp} y=e$, which is, furthermore, equivalent to $g^{\sharp} g x=x g^{\sharp} g$. Therefore $g^{\sharp} g \in C$; that is, $g \in G^{(C)}$.

Remark 3.7. In particular, when $C=C_{G}\left(G_{1}\right)$, our Theorem 3.2 gives useful information concerning the structure of the normaliser $\mathcal{N}_{G}\left(G_{1}\right)$. At the same time, for $\mathbb{K}$ algebraically closed, we have Theorem 2.2 as a special case; that is, $\mathrm{N}=G^{(C)}$ and $\mathrm{N}^{\prime}=G^{\left(C^{2}\right)}$, for $G, C, \mathrm{~N}$ and $\mathrm{N}^{\prime}$ as there. In other words, Theorem 3.2, which holds for many pairs of groups $\left(G, G_{1}\right)$, is a generalisation of Theorem 2.2. However, we would like to emphasise here that for the particular setting of $\left(G, G_{1}\right)$, with $G=\operatorname{SL}(m, \mathbb{K})$, we have more precise information concerning the normaliser $\mathrm{N}$. Namely, by a direct constructive argument we know that $\mathrm{N}^{\prime} \cong G_{1} \rtimes C^{2}$, the semidirect product of $G_{1}$ by $C^{2}$; see [S4, Theorem 0.1]. It turns out that the above isomorphism holds for some other pairs $\left(G, G_{1}\right)$, and so one would like to see whether a more general argument is available here. 
We are now ready to prove our main result.

Proof of Theorem 1.2. We have to prove the second part of the theorem; the first one is clear by the preceding lemma. For that purpose write $\boldsymbol{C}=C_{\boldsymbol{G}}\left(\boldsymbol{G}_{1}\right)$, and also $\boldsymbol{H}$ instead of $\boldsymbol{G}^{(\boldsymbol{C})}$. First notice that $\boldsymbol{H}=\mathcal{N}_{\boldsymbol{G}}\left(\boldsymbol{G}_{1}\right)$. We proceed as in the second half of the proof of the above lemma. Assume that $g \in \mathcal{N}_{\boldsymbol{G}}(\boldsymbol{H})$, and define $h=g^{\sharp} g$. By definition of the normaliser, for any $x \in \boldsymbol{G}_{1}$ we have $y=y(x)=g x g^{-1} \in \boldsymbol{H}$. And therefore $y^{\sharp} y=c(x)=c$, for some $c \in \boldsymbol{C}$. Furthermore, the last equality is equivalent to

$$
h x=c x h .
$$

The key observation, which we will prove below, is that necessarily $c=1$; see the proofs of [S3, Theorem 3.7(ii)] and [S4, Theorem 2.6(ii)], where it follows by elementary arguments. We then conclude that $h \in \boldsymbol{C}$; that is, $g \in \boldsymbol{H}$, and we are done.

To prove that $c=1$ we argue as follows. For $h$ as defined above, consider a map

$$
\phi_{h}: \boldsymbol{G}_{1} \rightarrow \boldsymbol{G}, \quad \phi_{h}(x)=[h, x] .
$$

Let $\imath_{1}: \boldsymbol{G}_{1} \rightarrow \boldsymbol{G}_{1}$ be the inversion map, $\imath_{1}(x)=x^{-1}$. We denote by $\mathcal{I}_{h}: \boldsymbol{G}_{1} \rightarrow \boldsymbol{G}$ the conjugation by $h$. Next, let $\Delta_{1}=\left\{(x, x) \mid x \in G_{1}\right\}$ be the diagonal of $G_{1} \times G_{1}$, and $\delta_{1}: G_{1} \rightarrow \Delta_{1}$ be the corresponding diagonal map. Finally, let $m: \boldsymbol{G} \times \boldsymbol{G} \rightarrow \boldsymbol{G}$ denote the multiplication map. If we define

$$
\begin{aligned}
& \mathcal{I}_{h} \times l_{1}: \boldsymbol{G}_{1} \times \boldsymbol{G}_{1} \rightarrow \boldsymbol{G} \times \boldsymbol{G}_{1}, \\
& \left(\mathcal{I}_{h} \times l_{1}\right)\left(x_{1}, x_{2}\right)=\left(\mathcal{I}_{h}\left(x_{1}\right), l_{1}\left(x_{2}\right)\right),
\end{aligned}
$$

then

$$
\phi_{h}=m \circ\left(\mathcal{I}_{h} \times l_{1}\right) \circ \delta_{1} .
$$

As a conclusion, $\phi_{h}$ is a morphism of affine varieties. We claim that it is, moreover, a homomorphism of algebraic groups. To see this, take arbitrary $x_{1}, x_{2} \in \boldsymbol{G}_{1}$. By (3.4) we have $c_{i}=c\left(x_{i}\right) \in \boldsymbol{C}$ so that $h x_{1}=c_{1} x_{1} h$ and $h^{-1} x_{2}^{-1}=x_{2}^{-1} h^{-1} c_{2}$. Hence we compute

$$
\begin{aligned}
\phi_{h}\left(x_{1} x_{2}\right) & =\left(h x_{1}\right) x_{2}\left(h^{-1} x_{2}^{-1}\right) x_{1}^{-1} \\
& =\left(c_{1} x_{1} h\right) x_{2}\left(x_{2}^{-1} h^{-1} c_{2}\right) x_{1}^{-1}=c_{1} c_{2}=\phi_{h}\left(x_{1}\right) \phi_{h}\left(x_{2}\right) .
\end{aligned}
$$

Observe now that (3.4), together with the fact that $C \leq \mathbb{K}^{\times}$, gives that $[h, x]=\lambda=$ $\lambda(x)$ for certain $\lambda \in \mathbb{K}^{\times}$. By taking the determinants of both sides of the last equality we obtain that $\lambda \in \boldsymbol{\mu}_{n}(\mathbb{K})$. As a consequence, $\operatorname{Im} \phi_{h} \subseteq \boldsymbol{D}$, where $\boldsymbol{D}=\boldsymbol{C} \cap \boldsymbol{\mu}_{n}(\mathbb{K})$. As $\boldsymbol{G}_{1}$ is connected, $\operatorname{Im} \phi_{h}$ is a connected subgroup as well. But $\boldsymbol{D}$ is discrete, and therefore $\phi_{h}$ is a constant map; that is, $\phi_{h}=1$. Thus we have proved our theorem.

REMARK 3.8. Concerning Theorem 1.2, notice the following interesting fact; see [S3, Theorem 3.7(ii)] for more details. Let $G=\operatorname{SL}(2 n, \mathbb{K})$, and let the map $A \mapsto A^{\sharp}$ be as in Section 2.3. Define $G_{1}=G^{(I)}$ as usual. Take $n=\varepsilon=1$ in particular, and consider the pair of groups $\left(G, G_{1}\right)$. It turns out that, for $\mathbb{K}=\overline{\mathbb{F}_{5}}, H=\mathcal{N}_{G}\left(G_{1}\right)$ is self-normalising in $G$. But for $\mathbb{K}=\mathbb{F}_{5}, \mathcal{N}_{G}(H) \neq H$. 
We proceed with an analogue of Lemma 3.6, which is again interesting in its own right. For this we need a little preparation; more details can be found in [S3, Section 1.2]. Let $\boldsymbol{G} \leq \mathrm{GL}(n, \overline{\mathbb{K}})$ again be a (connected) algebraic $\mathbb{K}$-group. We denote its Lie algebra by $(\mathfrak{5} \leq \mathfrak{g l}(n, \overline{\mathbb{K}})$. Suppose that we also have a connected closed subgroup $\boldsymbol{G}_{1} \leq \boldsymbol{G}$, and let $\boldsymbol{5}_{1}$ be its Lie algebra. Consider a pair $\left(G, G_{1}\right)$, where $G=\boldsymbol{G}(\mathbb{K}) \leq \mathrm{GL}(n, \mathbb{K})$ and $G_{1}=\boldsymbol{G}_{1}(\mathbb{K})$ are the corresponding groups of $\mathbb{K}$-points. We also consider a pair of Lie algebras $\left(\mathfrak{g}, \mathfrak{g}_{1}\right)$, where $\mathfrak{g}=\mathfrak{b}(\mathbb{K}) \leq \mathfrak{g l}(n, \mathbb{K})$ and $\mathfrak{g}_{1}=\mathfrak{G}_{1}(\mathbb{K})$ are corresponding $\mathbb{K}$-structures. Suppose that we have a map $A \mapsto A^{\sharp}$ satisfying $(\sharp \mathbf{1})$ and $(\sharp 2)$, and that both $G$ and $g$ are $\sharp$-stable. Furthermore, we suppose that $G$ operates on $g$ via the adjoint action. Also, we suppose that $G_{1}=G^{(I)}$ as before, and $\mathfrak{g}_{1}=\left\{X \in \mathfrak{g} \mid X^{\sharp}=-X\right\}$.

Lemma 3.9. Suppose that the setting and assumptions of the previous paragraph hold. Assume also that both $\mathcal{C}_{G}\left(\mathfrak{g}_{1}\right) \leq \mathcal{Z}(G)$ and (3.1) hold. Then

$$
\mathcal{N}_{G}\left(\mathfrak{g}_{1}\right)=\left\{g \in G \mid g^{\sharp} g \in C_{G}\left(\mathfrak{g}_{1}\right)\right\} .
$$

Proof. We proceed in a manner similar to the proof of Lemma 3.6. Namely, if we put $C=C_{G}\left(\mathfrak{g}_{1}\right)$, we must prove that $\mathcal{N}_{G}\left(\mathfrak{g}_{1}\right)=G^{(C)}$. Let us show only the inclusion from right to left. For that purpose take any $g \in G^{(C)}$. Let $X \in \mathfrak{g}_{1}$ be arbitrary, and define $Y=g X g^{-1}$. Using (3.1), one can immediately check that $Y \in \mathfrak{g}_{1}$. Hence $g \in \mathcal{N}_{G}\left(\mathfrak{g}_{1}\right)$.

Assume for the moment that $\mathbb{K}$ is algebraically closed of characteristic zero, and let $\boldsymbol{G}$ be a closed subgroup of $\mathrm{GL}(n, \mathbb{K})$. Let $\boldsymbol{G}_{1}$ be a closed subgroup of $\boldsymbol{G}$. Suppose that both $\boldsymbol{G}$ and $\boldsymbol{G}_{1}$ are connected. Let $\mathfrak{g}$ and $\mathfrak{g}_{1}$ be the Lie algebras of $\boldsymbol{G}$ and $\boldsymbol{G}_{1}$, respectively. Define $\mathbf{N}=\mathcal{N}_{\boldsymbol{G}}\left(\boldsymbol{G}_{1}\right)$. It is well known that $\mathbf{N}$ is a closed subgroup of $\boldsymbol{G}$, and its Lie algebra is equal to $N_{\mathfrak{g}}\left(\mathfrak{g}_{1}\right)$. It is also easy to see that

$$
\mathbf{N}=\mathcal{N}_{\boldsymbol{G}}\left(\boldsymbol{G}_{1}\right)=\mathcal{N}_{\boldsymbol{G}}\left(\mathfrak{g}_{1}\right)
$$

see, for example, [Bou, Ch. III, Section 9.4]. Concerning Lemmas 3.6 and 3.9, notice that for groups of rational points the analogue of (3.5) need not hold.

We conclude the present section with an easy proposition which contains some useful basic facts. Loosely speaking, it points in particular to the relationship between the classes of self-normalising (algebraic) subgroups and self-normalising Lie subalgebras.

Proposition 3.10. In the above setting and notation, suppose that $\mathfrak{g}_{1}$ is a selfnormalising subalgebra of $\mathfrak{g}$.

(i) $\mathbf{N} / \boldsymbol{G}_{1}$ is a finite group.

(ii) For an arbitrary closed subgroup $\boldsymbol{G}_{1} \leq \boldsymbol{H} \leq \mathbf{N}$, we have $\mathcal{N}_{\boldsymbol{G}}(\boldsymbol{H}) \leq \mathbf{N}$.

(iii) $\mathcal{N}_{\boldsymbol{G}}(\mathbf{N})=\mathbf{N}$; that is, $\mathbf{N}$ is a self-normalising subgroup of $\boldsymbol{G}$.

Proof. (i) This is clear by the fact that the Lie algebras of $\mathbf{N}$ and $\boldsymbol{G}_{1}$ are equal.

(ii) Let $g \in \mathcal{N}_{\boldsymbol{G}}(\boldsymbol{H})$ be arbitrary. For the morphism Int $g$, Int $g\left(\boldsymbol{G}_{1}\right)$ is a connected subgroup of $\boldsymbol{H}$. As $\boldsymbol{G}_{1}=\mathbf{N}^{o}=\boldsymbol{H}^{o}$, the connected components of the identity element $e_{G}$, we conclude that Int $g\left(\boldsymbol{G}_{1}\right)=\boldsymbol{G}_{1}$. Hence, $g \in \mathbf{N}$.

(iii) This follows by (ii). 


\section{Pairs of parabolic subgroups}

Recall the following well-known fact; see, for example, [Bor, Corollary 11.11].

Lemma 4.1. Let $\boldsymbol{G}$ be a connected linear algebraic group over $\mathbb{K}$. Suppose $\boldsymbol{B}$ is a Borel subgroup of $\boldsymbol{G}$. Then

$$
\mathcal{Z}(\boldsymbol{B})=\mathcal{Z}(\boldsymbol{G}) .
$$

The purpose of this section is to prove two results that in a certain sense complement the above lemma. Consider $G=\operatorname{GL}(n, \mathbb{K})$ or $\operatorname{SL}(n, \mathbb{K})$, where $n \geq 2$. Recall that the standard Borel subgroup $B$ of $G$ is the subgroup consisting of upper triangular matrices in $G$. A subgroup $Q_{s t}$ of $G$ is called standard parabolic if $Q_{s t}$ contains $B$. A subgroup $\bar{B}$ (respectively, $Q$ ) is called a Borel (respectively, parabolic) subgroup of $G$ if $\bar{B}$ (respectively, $Q$ ) is $G$-conjugate to $B$ (respectively, some standard parabolic). We have the following basic fact; in particular, it is an auxiliary result for Proposition 4.3 below.

Proposition 4.2. Suppose that $G$ is either $\operatorname{SL}(n, \mathbb{K})$, with $n>2$ or $\mathbb{K} \neq \mathbb{F}_{3}$, or $\operatorname{GL}(n, \mathbb{K})$. Let $Q$ be any parabolic subgroup of $G$. Then

$$
C_{G}(Q)=\mathcal{Z}(Q)=\mathcal{Z}(G) .
$$

For $G=\operatorname{SL}\left(2, \mathbb{F}_{3}\right)$ and its standard Borel subgroup $B$ we have $\mathcal{Z}(B)=B=C_{G}(B)$ and $\mathcal{Z}(G)=\{ \pm 1\}$.

Proof. Consider first the case $G=\mathrm{GL}(n, \mathbb{K})$. As will be seen below, we can assume that $Q=B$, the standard Borel subgroup. Therefore, let us first show that

$$
C_{G}(B)=\mathcal{Z}(B)=\mathcal{Z}(G),
$$

where of course $\mathcal{Z}(G)=\mathbb{K}^{\times}$. To see this, take some $A=\left(a_{i j}\right) \in G$ so that $A X=X A$, for all $X=\left(x_{i j}\right) \in B$. We will write the matrices $A X=\left(l_{i j}\right)$ and $X A=\left(r_{i j}\right)$. In particular, we have $l_{11}=r_{11}$, and hence $\sum_{i=2}^{n} x_{1 i} a_{i 1}=0$. If we take $x_{1 i} \neq 0$ and $x_{1 j}=0$ for all $j \neq i$, it further follows that $a_{i 1}=0$. Thus $a_{i 1}=0$ for all $i \neq 1$. Next, take $x_{12}=0$ and $x_{11}=x_{22}$ in $l_{12}=r_{12}$. Analogously, we deduce that $a_{i 2}=0$ for all $i>2$. Thus the equality $l_{12}=r_{12}$ becomes $a_{12}\left(x_{22}-x_{11}\right)=\left(a_{22}-a_{11}\right) x_{12}$. By taking

$$
x_{12}=0 \quad \text { and } \quad x_{11} \neq x_{22},
$$

we deduce that $a_{12}=0$, and then $a_{11}=a_{22}$. Suppose now that for $t \leq n$ we have shown $a_{i i}=\alpha$, for all $1 \leq i \leq t-1$, and $a_{k s}=0$ for all $1 \leq s<t$ and $1 \leq k \leq n, k \neq s$. Then we consider the equality $l_{1 t}=r_{1 t}$, which boils down to

$$
\alpha x_{1 t}+a_{1 t} x_{t t}=\sum_{i=1}^{n} x_{1 i} a_{i t} .
$$

By choosing $x_{1 t}=0$ and $x_{t t}=x_{11}$, (4.3) becomes $\sum_{i=2}^{n} x_{1 i} a_{i t}=x_{i t} a_{t t}$. Hence, as before, we have that $a_{k t}=0$ for all $2 \leq k \leq n$ and $k \neq t$. Thus (4.3) in fact becomes $a_{1 t}\left(x_{t t}-x_{11}\right)=\left(a_{t t}-\alpha\right) x_{1 t}$. As in (4.2), choose $x_{1 t}=0$ and $x_{t t} \neq x_{11}$. Then the above 
equality gives that $a_{1 t}=0$, and therefore $\alpha x_{1 t}=x_{1 t} a_{t t}$. Thus clearly $\alpha=a_{t t}$. In this way we have shown, by induction, that $A=\alpha \in \mathbb{K}^{\times}$, as we claimed.

Suppose now that $Q=Q_{s t}$ is a standard parabolic subgroup. By (4.1), and the inclusions $\mathcal{Z}(G) \subseteq \mathcal{C}_{G}(Q) \subseteq \mathcal{C}_{G}(B)$, we conclude that $C_{G}(Q)=\mathcal{Z}(G)$. Next, it is clear that also $\mathcal{Z}(G) \subseteq \mathcal{Z}(Q) \subseteq C_{G}(Q)$. Thus we have proved our proposition.

Now let $Q$ be conjugate to some standard parabolic $Q_{s t}$, via some $g \in G$; that is, $Q=g Q_{s t} g^{-1}$. Then take some $\bar{\gamma} \in G$, and define $\gamma=g^{-1} \bar{\gamma} g$. Clearly, $\bar{\gamma} \in C_{G}(Q)$ if and only if $\gamma \in C_{G}\left(Q_{s t}\right)$. As we have shown that $C_{G}\left(Q_{s t}\right)=\mathcal{Z}(G)$, it clearly follows that $C_{G}(Q)=\mathcal{Z}(G)$ as well. Thus we have again proved our proposition.

Now consider the case $G=\operatorname{SL}(n, \mathbb{K})$; here $\mathcal{Z}(G)=\boldsymbol{\mu}_{n}(\mathbb{K})$. Let $B$ again be the standard Borel subgroup of $G$. Suppose that $n>2$ or $\mathbb{K} \neq \mathbb{F}_{3}$. An easy inspection shows that the above proof of (4.1), and so of the proposition, works here as well. For this purpose it is crucial to observe that we can again ensure that we have (4.2) and the corresponding analogues.

The second claim of the proposition is clear.

For what follows the reader may consult [S3, Section 4], and in particular Lemma 4.2 there. For the moment let $\mathbb{K}$ be arbitrary, and $s=s_{n}$ be the $n \times n$ matrix having 1 on the skew diagonal and 0 elsewhere. Given $M \in M_{n}(\mathbb{K})$, define

$$
M^{\tau}=s M^{t} s
$$

that is, $M^{\tau}$ is the skew transpose of $M$. Let again $\varepsilon=-1$ or +1 . Then, analogously to Section 2.3, consider a map $A \mapsto A^{\sharp}=A_{\varepsilon}^{\sharp}$ on block matrices given by

$$
\left(\begin{array}{cc}
X & Y \\
Z & T
\end{array}\right)=A \longmapsto A^{\sharp}=\left(\begin{array}{cc}
T^{\tau} & \varepsilon Y^{\tau} \\
\varepsilon Z^{\tau} & X^{\tau}
\end{array}\right) .
$$

Of course, the map $A \mapsto A^{\sharp}$ defined above is not equal to the map $A \mapsto A^{\sharp}$ considered in Section 2.3. But we now also have exactly the same statement as in Lemma 2.1.

Now let $G=\operatorname{GL}(2 n, \mathbb{K})$ or $\operatorname{SL}(2 n, \mathbb{K})$, and let $B$ be the standard Borel subgroup of $G$. Obviously, $B$ is $\sharp$-stable. Define

$$
B_{1}=B_{1}^{\varepsilon}=\left\{g \in B \mid g^{\sharp} g=I\right\}=B \cap G_{1}^{\varepsilon},
$$

where

$$
G_{1}=G_{1}^{\varepsilon}=\left\{A \in G \mid A^{\sharp} A=I\right\} ;
$$

that is, $B_{1}$ is the standard Borel subgroup of $G_{1}$.

Analogously as before, any subgroup of $G_{1}$, which contains $B_{1}$, is called standard parabolic. A subgroup $Q_{1}$ of $G_{1}$ is called parabolic if it is $G_{1}$-conjugate to some standard parabolic.

For $G, G_{1}=G_{1}^{\varepsilon}, B$ and $B_{1}=B_{1}^{\varepsilon}$ as above, let $Q$ be some $\sharp$-stable standard parabolic subgroup of $G$. Define

$$
Q_{1}=Q_{1}^{\varepsilon}=\left\{g \in Q \mid g^{\sharp} g=I\right\}=Q \cap G_{1}^{\varepsilon} .
$$

Thus we have a pair of groups $\left(Q, Q_{1}\right)$. As we will see below, such pairs fit into our general setting. For that purpose we need an analogue of the previous proposition. 
Proposition 4.3. Let $\mathbb{K}$ be a field different from $\mathbb{F}_{3}$. Let $Q_{1}$ be any parabolic subgroup of $G_{1}$. Then

$$
C_{G}\left(Q_{1}\right)=\mathcal{Z}\left(Q_{1}\right)=\mathcal{Z}(G) .
$$

As a consequence, for pairs $\left(Q, Q_{1}\right)$ as above,

$$
C_{Q}\left(Q_{1}\right)=\mathcal{Z}\left(Q_{1}\right)=\mathfrak{Z}(Q)=\mathcal{Z}(G) .
$$

Proof. Suppose that $Q_{1}=g_{1} P_{1} g_{1}^{-1}$, where $P_{1} \supseteq B_{1}$ and $g_{1} \in G_{1}$. The same arguments as in the proof of Proposition 4.2 ensure the following: $C_{G}\left(B_{1}\right)=\mathcal{Z}(G)$ implies $C_{G}\left(P_{1}\right)=\mathcal{Z}(G)$; and $\mathcal{C}_{G}\left(P_{1}\right)=\mathcal{Z}(G)$ implies $\mathcal{C}_{G}\left(Q_{1}\right)=\mathcal{Z}(G)$. In other words, it is sufficient to prove the proposition for $Q_{1}=B_{1}$.

By way of preparation, first note the following easy observation which is formulated in a slightly more general form than we need below: a block matrix $A=\left(\begin{array}{ll}X & Y \\ 0 & T\end{array}\right)$ satisfies $A A^{\sharp}=I$ if and only if

$$
X T^{\tau}=I \quad \text { and } \quad Y X^{\tau}=-\varepsilon X Y^{\tau} .
$$

Let us now take, for example, $G=\operatorname{GL}(2 n, \mathbb{K})$. We will show that

$$
C_{G}\left(B_{1}\right)=\mathcal{Z}\left(B_{1}\right)=\mathcal{Z}(G)
$$

For that purpose, suppose that

$$
M=\left(\begin{array}{ll}
M_{11} & M_{12} \\
M_{21} & M_{22}
\end{array}\right) \in G
$$

such that $A M=M A$ for all $A \in B_{1}$. In particular, take $A=\left(\begin{array}{cc}I & Y \\ 0 & I\end{array}\right) \in B_{1}$, where $Y$ satisfies $Y=-\varepsilon Y^{\tau}$; see (4.4). Clearly, $A M=M A$ if and only if $Y M_{21}=M_{21} Y=0$ and $Y M_{22}=$ $M_{11} Y$. We treat the following three possibilities (see [S3, Claim 1, Theorem 3.7]).

(S) Symplectic case.

(O1) Orthogonal case, and $n$ odd.

(O2) Orthogonal case, and $n$ even.

For $(\mathbf{S})$ we take $Y=I$. Thus $M_{21}=0$ and $M_{22}=M_{11}$; that is, $M=\left(\begin{array}{cc}M_{11} & M_{12} \\ 0 & M_{11}\end{array}\right)$. Next, take $A=\left(\begin{array}{cc}X & 0 \\ 0 & \left(X^{-1}\right)^{\tau}\end{array}\right)$, where $X$ belongs to the standard Borel subgroup of $\operatorname{GL}(n, \mathbb{K})$. Now $A M=M A$ gives, in particular, that $X M_{11}=M_{11} X$. By Proposition 4.2 it follows that $M_{11}=\lambda I, \lambda \in \mathbb{K}^{\times}$. Finally, for $A=\left(\begin{array}{cc}X & Y \\ 0 & T\end{array}\right) \in B_{1}$ we have $A M=M A$ if and only if $X M_{12}=M_{12} T$. Then we choose $X=u I$, where $u \in \mathbb{K}$ is such that $u \neq u^{-1}$; here we use the fact that $\mathbb{K} \neq \mathbb{F}_{3}$. It follows that $M_{12}=0$. As a conclusion we have that $M=\lambda I$.

For (O2) we take $Y=\left(\begin{array}{cc}I_{r} & 0 \\ 0 & -I_{r}\end{array}\right)$, where $r=n / 2$; note that $Y^{\tau}=-Y$, as it must be. As $Y$ is regular, $Y M_{21}=0$ gives that $M_{21}=0$. Next, for $M=\left(\begin{array}{cc}M_{11} & M_{12} \\ 0 & M_{22}\end{array}\right)$ and $A$ chosen as in $(\mathbf{S})$, the same argument as there gives that $M_{11}=\lambda_{1} I$ and $M_{22}=\lambda_{2} I$, for certain $\lambda_{1}, \lambda_{2} \in \mathbb{K}^{\times}$. But $Y M_{22}=M_{11} Y$ implies that $\lambda_{1}=\lambda_{2}=\lambda$. It remains to note, as in $(\mathbf{S})$, that $M_{12}=0$.

For (O1) consider a block-diagonal matrix

$$
Y=\operatorname{diag}\left(I_{r-1}, 0,-I_{r-1}\right),
$$


where $2 r=n+1$. As $Y M_{21}=M_{21} Y=0$, it follows that $M_{21}=q E_{r r}$, for some $q \in \mathbb{K}$. Again take $A$ as in $(\mathbf{S})$, where $X=\left(x_{i j}\right)$ is an upper triangular regular matrix such that $x_{r r}^{2} \neq 1$; such $X$ can be chosen when the field $\mathbb{K}$ has at least four elements. As $M A=A M$ implies, in particular, that $X^{\tau} M_{21} X=M_{21}$, an easy calculation shows that necessarily $q=0$; that is, $M_{21}=0$. The rest of the argument is the same as for (O2).

Thus we have proved our proposition.

\section{References}

[Bor] A. Borel, Linear Algebraic Groups, second enlarged edition, Graduate Texts in Mathematics, 126 (Springer, New York, 1997).

[Bou] N. Bourbaki, Lie Groups and Lie Algebras (Springer, Berlin, 1989), Ch. 1-3.

[BK] R. Brylinski and B. Kostant, 'Nilpotent orbits, normality, and Hamiltonian group actions', J. Amer. Math. Soc. 7 (1994), 269-298.

[Ko1] T. Kobayashi, 'Discrete decomposability of the restriction of $A_{\mathfrak{q}}(\lambda)$ with respect to reductive subgroups III. Restriction of Harish-Chandra modules and associated varieties', Invent. Math. 131 (1998), 229-256.

[Ko2] T. Kobayashi, 'Discretely decomposable restrictions of unitary representations of reductive Lie groups-examples and conjectures', in: Analysis on Homogeneous Spaces and Representation Theory of Lie Groups, Okayama-Kyoto (1997), Advanced Studies in Pure Mathematics, 26 (Math. Soc. Japan, Tokyo, 2000), pp. 99-127.

[Ko3] T. Kobayashi, 'Restrictions of generalized Verma modules to symmetric pairs', Transform. Groups 17 (2012), 523-546.

[Kob] N. Koblitz, A Course in Number Theory and Cryptography, 2nd edn, Graduate Texts in Mathematics, 114 (Springer, Berlin, 1994).

[Ks] B. Kostant, 'A branching law for subgroups fixed by an involution and a noncompact analogue of the Borel-Weil theorem', in: Noncommutative Harmonic Analysis, Progress in Mathematics 220 (Birkhäuser, Boston, 2004), pp. 291-353.

[LS] T. Levasseur and S. P. Smith, 'Primitive ideals and nilpotent orbits in type $G_{2}$ ', J. Algebra 114 (1988), 81-105.

[O1] A. A. Osinovskaya, 'Restrictions of irreducible representations of classical algebraic groups to root $A_{1}$-subgroups', Comm. Algebra 31 (2003), 2357-2379.

[O2] A. A. Osinovskaya, 'On the restrictions of modular irreducible representations of algebraic groups of type $A_{n}$ to naturally embedded subgroups of type $A_{2}$ ', J. Group Theory 8 (2005), 43-92.

[S1] B. Širola, 'Pairs of semisimple Lie algebras and their maximal reductive subalgebras', Algebr. Represent. Theory 11 (2008), 233-250.

[S2] B. Širola, 'Pairs of Lie algebras and their self-normalizing reductive subalgebras', J. Lie Theory 19 (2009), 735-766.

[S3] B. Širola, 'Normalizers and self-normalizing subgroups', Glas. Mat. Ser. III 46 (2011), 385-414.

[S4] B. Širola, 'Normalizers and self-normalizing subgroups II', Cent. Eur. J. Math. 9 (2011), $1317-1332$.

\section{BORIS ŠIROLA, Department of Mathematics, University of Zagreb, Bijenička 30,}

10000 Zagreb, Croatia

e-mail: sirola@math.hr 